\title{
DIVERGENCE OF AVERAGES OBTAINED BY SAMPLING A FLOW
}

\author{
MUSTAFA AKCOGLU, ALEXANDRA BELLOW, \\ ANDRES DEL JUNCO, AND ROGER L. JONES
}

(Communicated by Andrew M. Bruckner)

\begin{abstract}
In this paper we consider ergodic averages obtained by sampling at discrete times along a measure preserving ergodic flow. We show, in particular, that if $U_{t}$ is an aperiodic flow, then averages obtained by sampling at times $n+t_{n}$ satisfy the strong sweeping out property for any sequence $t_{n} \rightarrow 0$. We also show that there is a flow (which is periodic) and a sequence $t_{n} \rightarrow 0$ such that the Cesaro averages of samples at time $n+t_{n}$ do converge a.e. In fact, we show that every uniformly distributed sequence admits a perturbation that makes it a good Lebesgue sequence.
\end{abstract}

Let $(X, \Sigma, m, U)$ denote a dynamical system, where $(X, \Sigma, m)$ is a probability space and $U=\left\{U_{t}: t \in R\right\}$ is an ergodic measure preserving flow acting on $X$. Let $\left(n_{k}\right)_{k=1}^{\infty}$ be any increasing sequence of integers and let $\left(t_{k}\right)_{k=1}^{\infty}$ be a sequence of numbers converging to zero. Define the averaging operators $C_{N}$ by

$$
C_{N} f(x)=\frac{1}{N} \sum_{k=1}^{N} f\left(U_{n_{k}+t_{k}} x\right) .
$$

We will be concerned with the almost everywhere convergence properties of this sequence of operators.

If the sequence $\left(t_{k}\right)_{k=1}^{\infty}$ is chosen to be linearly independent over the rationals, then using Bourgain's entropy method, Bergelson, Boshernitzan, and Bourgain showed that these averages can diverge a.e. even for $f \in L^{\infty}$ [4]. In fact, using a result of Rosenblatt [9], their result implies that in the case of an aperiodic flow there is a positive constant $\delta$, which depends only on the sequence $\left(n_{k}+t_{k}\right)$, such that given $\varepsilon>0$, we can take $f=\chi_{A}$ for some set $A$ with $m(A)<\varepsilon$ and $\lim \sup C_{N} f(x)>\delta$ a.e.

In what follows we shall consider the circle group $[0,1)(\bmod 1)$. When there is no ambiguity, we shall simply write $[0,1)$.

Throughout the paper we will use the notation $\{x\}$ to denote the fractional part of $x$, i.e., $\{x\}=x-[x]$. Lebesgue measure of a set $A$ will be denoted by $\mu(A)$.

Received by the editors October 2, 1991.

1991 Mathematics Subject Classification. Primary 28D10; Secondary 26D15.

Key words and phrases. Ergodic flow, good Lebesgue sequences, uniform distribution.

The work of A. Bellow and R. L. Jones is partially supported by NSF Grant DMS-8910947. 
A sequence of operators, $M_{k}$, on a probability space is said to have the "strong sweeping out property" if given $\varepsilon>0$, there exist a set $A$, with $m(A)<$ $\varepsilon$, such that $\lim \sup M_{k} \chi_{A}=1$ a.e. and $\lim \inf M_{k} \chi_{A}=0$ a.e.

Clearly a sequence of operators that satisfies the "strong sweeping out property" is very badly behaved.

Using results by Jones and Wierdl [7], it is easy to see that in the special case when $\left(t_{k}\right)$ are linearly independent over the rationals, the averaging operators $C_{N}$ have the "strong sweeping out property". However, much more is true!

Theorem 1. For any sequence $\left(t_{k}\right)_{k=1}^{\infty}$ that converges to zero and any aperiodic flow, the averaging operators $C_{N}$ have the strong sweeping out property.

The proof of this fact will consist of first proving a local ergodic theorem on a special flow on the circle group $[0,1)(\bmod 1)$, then proving the failure of a maximal inequality on an interval $[0, L) \subset R$, with translation, and finally a transference of this maximal function property to the setting of a general flow via a continuous version of the Rohlin lemma. We begin with the following local ergodic theorem.

Theorem 2. Let $\left(t_{k}\right)_{k=1}^{\infty}$ be any sequence that converges to zero, and define the averaging operators $T_{N}$ by $T_{N} f(x)=\frac{1}{N} \sum_{k=1}^{N} f\left(\left\{x+t_{k}\right\}\right)$ for $f:[0,1) \rightarrow$ $[0,1)$. Then the operators $T_{N}$ have the strong sweeping out property.

Remark. Weaker versions of this lemma are known. Bourgain [5] showed that these operators have unbounded metric entropy and hence fail to converges a.e. even for $f \in L^{\infty}$. Rosenblatt [9] noticed that this implies " $\delta$ sweeping out". Akcoglu, del Junco, and Lee [1] proved " $\delta$ sweeping out" without using the entropy method. In [3] the methods of Akcoglu, del Junco, and Lee are used to prove that in fact we have strong sweeping out. We refer the interested reader to that paper for the proof.

Theorem 3. Let $\left(t_{k}\right)_{k=1}^{\infty}$ converge to zero and let $\left(n_{k}\right)_{k=1}^{\infty}$ be any increasing sequence of integers. Define the operators

$$
\widetilde{T}_{N} f(t)=\frac{1}{N} \sum_{k=1}^{N} f\left(t+n_{k}+t_{k}\right)
$$

for $f: R \rightarrow R$. Then for every $\varepsilon>0$ and $K>0$, there is an $L>0$ and a set $A \subset[0, L]$ that satisfies

(a) $\mu(A) / L<\varepsilon$ and

(b) $\mu\left(\left\{t \in[0, L): \sup _{n>K} \widetilde{T}_{n} \chi_{A}(t)>1-\varepsilon\right\}\right)>L(1-\varepsilon)^{2}$.

(Here $\mu(A)$ denotes the Lebesgue measure of the set $A$.)

Proof. We can assume without loss of generality that $\left|t_{k}\right|<1$. First note that given $\varepsilon>0$ we can find a set $E$ such that $\mu(E)<\varepsilon$, but such that the operators $T_{N}$ have the strong sweeping out property on $[0,1)(\bmod 1)$. Define the set $P$ by $\chi_{P}=\chi_{E}$ on $[0,1)$ and extend $P$ so that $\chi_{P}$ is periodic with period 1 on 
all of $R$. Define $\chi_{A}=\chi_{P} \chi_{[0, L)}$. Consequently if $t \in\left[1, L-n_{N}-1\right)$ then

$$
\begin{aligned}
\widetilde{T}_{N} \chi_{A}(t) & =\frac{1}{N} \sum_{k=1}^{N} \chi_{A}\left(t+n_{k}+t_{k}\right)=\frac{1}{N} \sum_{k=1}^{N} \chi_{A}\left(t+t_{k}\right) \\
& =\frac{1}{N} \sum_{k=1}^{N} \chi_{A}\left(\left\{t+t_{k}\right\}\right)=\frac{1}{N} \sum_{k=1}^{N} \chi_{E}\left(\left\{t+t_{k}\right\}\right)=T_{N} \chi_{E}(x) .
\end{aligned}
$$

Because we know $\sup _{N>K} T_{N} \chi_{E}=1$ a.e. on $[0,1)(\bmod 1)$, we can find an integer $M$ so that

$$
\mu\left(\left\{\sup _{K<N \leq M} T_{N} \chi_{E}>1-\varepsilon\right\}\right)>1-\varepsilon .
$$

Select $L$ so large that $\left(L-n_{M}-2\right) / L>1-\varepsilon$. Consequently, we have

$$
\begin{gathered}
\mu\left(\left\{t \in\left[1, L-n_{M}-1\right): \sup _{K<N \leq M} \tilde{T}_{N} \chi_{A}>1-\varepsilon\right\}\right) \\
>(1-\varepsilon)\left(L-n_{M}-2\right)>(1-\varepsilon)^{2} L .
\end{gathered}
$$

Thus the set $A$ satisfies the conditions required for Theorem 3 .

Proof of Theorem 1. Theorem 1 now follows from Theorem 2 and an application of a continuous version of the Rohlin tower construction. From the Rohlin tower construction [8] we know that given $\varepsilon>0$ and $L$, there is a set $B$ such that

(i) the sets $U_{t} B$ are disjoint for $t, 0 \leq t<L$, and $Y=\bigcup_{0 \leq t<L} U_{t} B$ is measurable in $X$.

(ii) $m\left(\bigcup_{0 \leq t<L} U_{t} B\right)>1-\varepsilon$.

(iii) Let $Q$ be the interval $[0, L)$ with Lebesgue measure $\mu$ and let $\varphi: B \times Q \rightarrow Y$ be the natural bijection defined by $\varphi(x, t)=U_{t} x$. Then there is a measure $m_{B}$ on the set $B$ (which is singular with respect to the given measure $m$ on $X$ ) such that both $\varphi$ and $\varphi^{-1}$ are measurable measure-preserving maps between $\left(B \times Q, m_{B} \times \mu\right)$ and $(Y, m)$. In particular,

$$
1-\varepsilon<\int_{B} L d m_{B}(x)<1 .
$$

Let $F=X-\bigcup_{0 \leq t<L} U_{t} B$.

Define a function $f: X \rightarrow X$ as follows: on each "fiber"

$$
f\left(U_{t} x\right)=\chi_{A}(t) \text { for } 0 \leq t<L \text { and } x \in B,
$$

and $f=0$ on $F$. We have

$$
\begin{aligned}
\|f\|_{1} & =\int_{X} f(x) d m(x)=\int_{Y} f(y) d m(y)+\int_{F} f(x) d m(x) \\
& =\int_{B} \int_{0}^{L} f\left(U_{t} x\right) d \mu(t) d m_{B}(x) \leq \int_{B} \varepsilon L d m_{B}(x)<\varepsilon,
\end{aligned}
$$


and we have

$$
\begin{aligned}
& m\left(\left\{y \in Y: \sup _{K<N \leq M} C_{N} f(y)>1-\varepsilon\right\}\right) \\
& \geq \int_{B} \int_{1}^{L-n_{M}-1} \chi_{\left\{\sup _{K<N \leq M} \widetilde{T}_{N} \chi_{A}>1-\varepsilon\right\}}(t) d t d m_{B}(x) \\
& \geq \int_{B}(1-\varepsilon)^{2} L d m_{B}(x)>(1-\varepsilon)^{2}(1-\varepsilon)=(1-\varepsilon)^{3} .
\end{aligned}
$$

We can now apply a well-known result of del Junco and Rosenblatt [6] that says that if for each $\varepsilon>0$ and $K$, we can find a set $A$ such that $\sup _{n>K} C_{n} \chi_{A}>1-\varepsilon$ on a set of measure at least $1-\varepsilon$, then the sequence $C_{n}$ has the "strong sweeping out property".

In order to make the above argument work, it is important to have an aperiodic transformation. Otherwise it is not possible to make the necessary Rohlin tower. In fact, in the periodic case, Theorem 1 is false. We have the following result.

Theorem 4. Let $(X, \Sigma, m, U)$ be the dynamical system consisting of the unit interval with Lebesgue measure and $U$ the measure preserving ergodic flow $U_{t} x=x+\theta t \bmod 1$, with $\theta$ irrational. For this dynamical system we can find a sequence $\left(p_{k}\right)_{k=1}^{\infty}$ with the property that $\lim _{k \rightarrow \infty} p_{k}=0, p_{k} \neq 0$ for any $k$, but such that the averages

$$
\lim _{N \rightarrow \infty} \frac{1}{N} \sum_{k=1}^{N} f\left(U_{k+p_{k}} x\right)
$$

converge a.e. for all $f \in L^{1}(X)$.

Theorem 4 will be a special case of the following theorem, which in a certain sense provides an answer to a question raised in [2].

Theorem 5. Let $\left(s_{n}\right)_{n=0}^{\infty}$ be uniformly distributed in $[0,1)$. Then there is a sequence $\left(\varepsilon_{n}\right)_{n=0}^{\infty}, \varepsilon_{n} \neq 0$ for all $n, \varepsilon_{n} \rightarrow 0$, such that $\left(s_{n}+\varepsilon_{n}\right)_{n=0}^{\infty}$ is good Lebesgue, i.e., for all $f \in L^{1}$,

$$
\frac{1}{N} \sum_{n=0}^{N} f\left(\left\{x+s_{n}+\varepsilon_{n}\right\}\right) \rightarrow \int f d \mu \text { a.e. }
$$

We divide the proof into several lemmas.

Definition 1. The $n$-tuple $\left(u_{0}, u_{1}, \ldots, u_{n-1}\right)$ is called $2^{m}$-good if for all dyadic intervals of order $2^{m}, I=\left[r / 2^{m}, s / 2^{m}\right)$,

$$
\left|\frac{1}{n} \sum_{i=0}^{n-1} \chi_{I}\left(u_{i}\right)-\mu(I)\right|<\frac{1}{2^{m+1}} .
$$

Lemma 1. Let $\left(x_{n}\right)_{n=0}^{\infty}$ be uniformly distributed mod 1. For each integer $m \geq 1$, there exists $n_{1} \geq 1$ such that $n \geq n_{1}$ implies $\left(x_{n}, x_{n+1}, \ldots, x_{2 n-1}\right)$ is $2^{m}-$ good.

Proof. This follows easily from the definitions. 
Lemma 2. Suppose $n=k 2^{m}$ and that the $n$-tuple $\left(u_{0}, u_{1}, \ldots, u_{n-1}\right)$ is $2^{m}$-good. Let $\left(u_{0}^{1}, u_{1}^{1}, \ldots, u_{n-1}^{1}\right)$ be a nondecreasing rearrangement of $\left(u_{0}, u_{1}, \ldots, u_{n-1}\right)$. Then

$$
\left|u_{i}^{1}-\left[\frac{i}{k}\right] \frac{1}{2^{m}}\right|<\frac{2}{2^{m}}
$$

Proof. For $0<j<2^{m}$, note that

$$
\sum_{i=0}^{n-1} \chi_{\left[0, j / 2^{m}\right)}\left(u_{i}\right) \geq n\left(\frac{j}{2^{m}}-\frac{1}{2^{m+1}}\right)=k j-\frac{k}{2}>k j-k=(j-1) k
$$

and, similarly,

$$
\sum_{i=0}^{n-1} \chi_{\left[0, j / 2^{m}\right)}\left(u_{i}\right)<(j+1) k
$$

thus

whence

$$
u_{(j-1) k}^{1}<\frac{j}{2^{m}} \quad \text { and } \quad u_{(j+1) k}^{1} \geq \frac{j}{2^{m}}
$$

It follows that for $0 \leq r<k$,

$$
\frac{j-1}{2^{m}} \leq u_{j k}^{1}<\frac{j+1}{2^{m}}
$$

$$
\frac{j-1}{2^{m}} \leq u_{j k+r}^{1} \leq u_{(j+1) k}^{1}<\frac{j+2}{2^{m}}
$$

and for $i=j k+r,[i / k]=j$. Note also that for $j=1$,

$$
0 \leq u_{k}^{1}<\frac{1+1}{2^{m}}=\frac{2}{2^{m}}
$$

hence for $0 \leq r<k,[r / k]=0$ and $u_{r}^{1} \leq 2 / 2^{m}$. This finishes the proof.

Lemma 3. Suppose $n=k 2^{m}$ and the $n$-tuples $\left(u_{0}, u_{1}, \ldots, u_{n-1}\right)$ and $\left(v_{0}, v_{1}, \ldots, v_{n-1}\right)$ are $2^{m}$-good. Then there exists a permutation $\tau$ of $\{0,1$, $\ldots, n-1\}$ such that $\left|u_{i}-v_{\tau(i)}\right|<4 / 2^{m}$ for $i=0,1, \ldots, n-1$.

Proof. This result follows immediately from Lemma 2 , applied to $\left(u_{0}, u_{1}\right.$, $\left.\ldots, u_{n-1}\right)$ and $\left(v_{0}, v_{1}, \ldots, v_{n-1}\right)$.

Lemma 4. Let $\left(u_{n}\right)$ be a sequence in $[0,1)$ and define

$$
A_{N} f(x)=\frac{1}{N} \sum_{i=0}^{N-1} f\left(\left\{x+u_{i}\right\}\right) \text { for } f \in L^{1} .
$$

Suppose that $N_{1}<N_{2}<\cdots$ satisfies $N_{i+1}<D N_{i}$ for some constant $D>0$ and that there is a maximal inequality

$$
\mu\left(\left\{\sup _{i} A_{N_{i}} f>\lambda\right\}\right) \leq \frac{c}{\lambda}\|f\|_{1} \quad \text { for } f \in L_{+}^{1} .
$$

Then

$$
\mu\left(\left\{\sup _{N} A_{N} f>\lambda\right\}\right) \leq \frac{c D}{\lambda}\|f\|_{1} \quad \text { for } f \in L_{+}^{1} .
$$

Proof. This is easy and well known. 
Proof of Theorem 5. First choose an irrational $\alpha \in[0,1)$ such that the sets $\{\{(n+1) \alpha\}: n=0,1, \ldots\}$ and $\left\{s_{m}: m=0,1, \ldots\right\}$ are disjoint. (This is possible since there are at most countably many $\alpha$ 's for which $\{\{(n+1) \alpha\}$ : $n=0,1, \ldots\} \cap\left\{s_{m}: m=0,1, \ldots\right\} \neq \varnothing$.) Set $t_{n}=\{(n+1) \alpha\}$. Now define the increasing sequence of integers

$$
\begin{aligned}
N_{1}^{(1)} & <N_{2}^{(1)}<\cdots<N_{k_{1}}^{(1)}<N_{1}^{(2)}<N_{2}^{(2)}<\cdots<N_{k_{2}}^{(2)} \\
& <\cdots<N_{1}^{(m)}<N_{2}^{(m)}<\cdots<N_{k_{m}}^{(m)}<\cdots
\end{aligned}
$$

as follows:

If $m \geq 1$ and $1 \leq j<k_{m}$ then $N_{j+1}^{(m)}=2 N_{j}^{(m)}$, and if $j=k_{m}$ then $N_{1}^{(m+1)}=2 N_{k_{m}}^{(m)}$.

Choose $N_{1}^{(1)}=n_{0}$, an even integer with the property that

$$
\begin{array}{ll}
n \geq n_{0} \text { implies } & \left(s_{n}, s_{n+1}, \ldots, s_{2 n-1}\right) \text { is } 2^{1} \text {-good and } \\
& \left(t_{n}, t_{n+1}, \ldots, t_{2 n-1}\right) \text { is } 2^{1} \text {-good. }
\end{array}
$$

Assume $N_{1}^{(m)}$ is defined and $N_{i+1}^{(m)}=2 N_{i}^{(m)}$ and choose $k_{m} \geq m+1$ large enough that for $N_{1}^{(m+1)}=2 N_{k_{m}}^{(m)}=2^{k_{m}} N_{1}^{(m)}$ we have

$$
\begin{array}{ll}
n \geq N_{1}^{(m+1)} \text { implies } & \left(s_{n}, s_{n+1}, \ldots, s_{2 n-1}\right) \text { is } 2^{(m+1)} \text {-good and } \\
& \left(t_{n}, t_{n+1}, \ldots, t_{2 n-1}\right) \text { is } 2^{(m+1)} \text {-good. }
\end{array}
$$

For $m \geq 1$ and $1 \leq j \leq k_{m}$ define the block of consecutive integers $B_{j}^{(m)}$ of length $N_{j}^{(m)}$ :

$$
B_{j}^{(m)}=\left\{N_{j}^{(m)}, N_{j}^{(m)}+1, N_{j}^{(m)}+2, \ldots, 2 N_{j}^{(m)}-1\right\} .
$$

By Lemma 3, there is a permutation $\tau\left(=\tau_{j}^{(m)}\right)$ of the block $B_{j}^{(m)}$ such that

$$
\left|t_{i}-s_{\tau(i)}\right|<4 / 2^{m} \quad \text { for } i \in B_{j}^{(m)} \text {. }
$$

With $\varepsilon_{\tau(i)}=t_{i}-s_{\tau(i)}$, we have

$$
\varepsilon_{i} \neq 0 \text { and } \quad\left|\varepsilon_{i}\right| \leq 4 / 2^{m} \quad \text { for all } i \in B_{j}^{(m)} \text {. }
$$

For $m \geq 1$ and $1 \leq j \leq k_{m}$, note that if $f \in L^{1}$ then

$$
\sum_{n=n_{0}}^{N_{j}^{(m)}-1} f\left(x+s_{n}+\varepsilon_{n}\right)=\sum_{n=n_{0}}^{N_{j}^{(m)}-1} f\left(x+t_{n}\right)
$$

(since we are summing on complete consecutive blocks $B_{q}^{(p)}$ and on each one of these blocks the sum is independent of the order of summation); note also that because of (1), we have $\varepsilon_{n} \rightarrow 0$.

Because of (2), an application of Lemma 4 now yields the maximal inequality for the averages

$$
A_{N} f(x)=\frac{1}{N} \sum_{n=0}^{N-1} f\left(x+s_{n}+\varepsilon_{n}\right), \quad f \in L^{1} .
$$


Since the sequence $\left(s_{n}+\varepsilon_{n}\right)$ is also uniformly distributed $\bmod 1$, there is also a dense class-namely, the continuous functions on $[0,1)$-for which a.e. convergence obtains. This completes the proof.

\section{REFERENCES}

1. M. Akcoglu, A. del Junco, and W. Lee, A solution to a problem of A. Bellow, Almost Everywhere Convergence II, Proceedings of the International Conference on Almost Everywhere Convergence in Probability and Ergodic Theory (A. Bellow and R. Jones, eds.), Academic Press, New York, 1991.

2. A. Bellow, Two problems, Proc. Oberwolfach Conference in Measure Theory (June 1981), Lecture Notes in Math., vol. 945, Springer-Verlag, Berlin and New York, 1982.

3. M. Akcoglu, A. Bellow, R. Jones, V. Losert, K. Reinhold, and M. Wierdl, The strong sweeping out property for lacunary sequences, for the Riemann sums, and related matters, preprint.

4. V. Bergelson, M. Boshernitzan, and J. Bourgain, Some results on non-linear recurrence, preprint.

5. J. Bourgain, Almost sure convergence and bounded entropy, Israel J. Math. 73 (1988), 79-97.

6. A. del Junco and J. Rosenblatt, Counter examples in ergodic theory and number theory, Math. Ann. 247 (1984), 185-197.

7. R. Jones and M. Wierdl, Convergence and divergence of ergodic averages, preprint.

8. D. Lind, Locally compact measure preserving flows, Adv. in Math. 15 (1975), 175-193.

9. J. Rosenblatt, Universally bad sequences in ergodic theory, Almost Everywhere Convergence II, Proceedings of the International Conference on Almost Everywhere Convergence in Probability and Ergodic Theory (A. Bellow and R. Jones, eds.), Academic Press, New York, 1991.

(M. Akcoglu and A. del Junco) Department of Mathematics, University of Toronto, TORONTO, CANADA

E-mail address, M. Akcoglu: akcoglu@math.toronto.edu

E-mail address, A. del Junco: deljunco@math.toronto.edu 60201

(A. Bellow) Department of Mathematics, Northwestern University, Evanston, Illinois

(R. L. Jones) Department of Mathematics, DePaul University, 2219 North Kenmore, Chicago, Illinois 60614

E-mail address: matrlj@depaulo.bitnet 